\title{
The Homological Conjectures
}

\author{
Paul C. Roberts
}

\begin{abstract}
We describe the conjectures in Commutative Algebra known as the Homological Conjectures, outline their history over the past forty years or so, and give a summary of their current status, including a list of the main conjectures that are open at the present time.
\end{abstract}

Keywords. Homological Conjectures, Multiplicities, Cohen-Macaulay Modules, Direct Summand Conjecture.

2010 Mathematics Subject Classification. Primary: 13D22, 13D02; Secondary: 13C10, 13C14, 13H05, 14C17.

\section{Introduction}

The term "Homological Conjectures" is used here to refer to a certain set of related conjectures about homological properties of commutative rings. While there are numerous conjectures in this area, the ones discussed here are those collected in a monograph of Mel Hochster in 1975 entitled "Topics in the homological theory of modules over commutative rings" [24], as well as several ones that have developed out of them. In this monograph Hochster stated a number of earlier conjectures, added a few of his own, and solved several of them. Since then new ones have been added and some of them have been settled. It is the aim of this article to outline this history, starting at the beginning and ending by giving an idea of the present situation. We will attempt to give some idea of the methods and concepts behind the various advances, and give references to more complete accounts.

The article is organized as follows. In each of the first few sections we discuss a set of related conjectures on Hochster's diagram and follow their development up to the present. These sections follow a roughly chronological order as far as the origins of the conjectures are concerned, beginning with Serre's multiplicity conjectures which were one of the major influences behind the whole subject. However, there has been recent progress even on some of the earliest conjectures, and we will discuss, for example, recent developments on Serre's original conjectures before getting to generalizations of these conjectures which came much earlier. In addition to the major advances, we will mention many other developments, but there are a lot of them and we have not attempted to cover them all. 
We give Hochster's 1975 diagram below. The conjectures appearing in the diagram will then be stated in the following sections. We give a table of contents below, including the numbers from the diagram that are defined in each section. Those denoted $\mathrm{M}_{0}$, $\mathrm{M}_{1}$, and $\mathrm{M}_{2}$ are parts of (8), the Serre Multiplicity Conjectures, and (9), the Strong Multiplicity Conjectures.

Here is the outline.

(i) The Serre Multiplicity Conjectures ((1), (8)).

(ii) The Peskine-Szpiro Intersection Conjecture ((2), (3), (4), (5)).

(iii) Generalizations of the Multiplicity Conjectures ((9), (12), (13)).

(iv) The Monomial, Direct Summand, and Canonical Element Conjectures ((10), (11))

(v) Cohen-Macaulay Modules and Algebras ((6), (7)).

(vi) The Syzygy Conjecture and the Improved New Intersection Conjecture.

(vii) Tight Closure Theory

(viii) The Strong Direct Summand Conjecture.

(ix) Almost Cohen-Macaulay Algebras.

(x) A Summary of Open Questions.

There have been several summaries of progress on these conjectures over the years, including two in the last decade. Jan Strooker [63] has an book on the state of the Homological Conjectures in 1990; it also includes a lot of the necessary background in Commutative Algebra. There is also a set of notes coming from a Minicourse on Classical Questions in Commutative Algebra at the University of Utah which covered many aspects of the subject. These notes can be found at the University of Utah website at http://www.math.utah.edu/vigre/minicourses/2004.html\#b. Hochster also has a summary from the conference in honor of Phil Griffith which talks about some of the recent developments [30].

We next give the diagram of implications between various conjectures from Hochster's monograph from 1975. The conjectures will be stated in detail in subsequent sections. Implications are indicated in the diagram by arrows, and the diagram is set up so that, for the most part, the stronger conjectures are toward the top and the left side. Since 1975 some of the conjectures have been proven or counterexamples have been found, and in addition some new implications have been found and new conjectures added to the list. We will give a more recent version, with conjectures which have been settled taken off the list and new ones added, later in the paper. 
(1) Rigidity

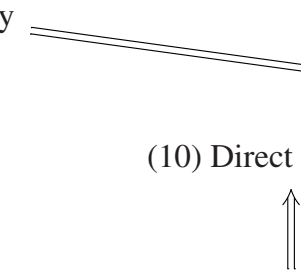

(11) Monomial

(3) Intersection

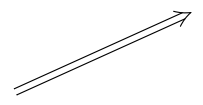

(2) Zero Divisor

(6) Small C-M
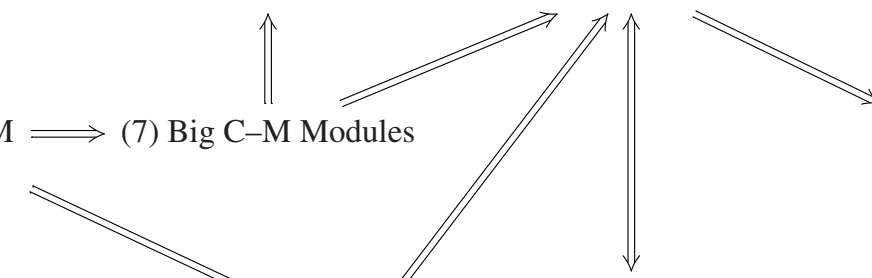

Modules

$>(7)$

Big C-M Modules

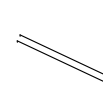

(4) Homological

Height

(12) Strong Intersection
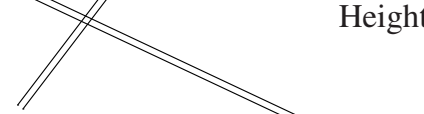

^

$\mathrm{M}_{0}$ of (9)+(13)

Codimension

$\left(\mathrm{M}_{1} \Rightarrow \mathrm{M}_{2}\right)$,

$\Downarrow$

$\mathrm{M}_{0}$ of (9)

(9) Strong Multiplicities

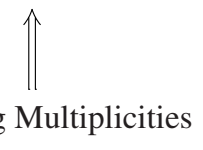

regular case

Figure 1. Hochster's diagram of homological conjectures in 1975.

\section{The Serre Multiplicity Conjectures}

Among the earliest conjectures in this subject were those of Jean-Pierre Serre which arose from his theory of intersection multiplicities using homological methods. The idea was to extend the algebraic theory from classical methods that worked, say, for intersections of curves in the plane, to a more general situation.

We look briefly at the case of the intersection of two curves in the affine plane over an algebraically closed field $k$. In this case each curve is defined by one polynomial in two variables, say $x$ and $y$, so we have polynomials $f$ and $g$ defining the two curves. The condition that a point $p$ in the plane corresponding to a maximal ideal $\mathfrak{m}$ 
of $A=k[x, y]$ is an isolated point of intersection means that the ideal $(f, g)$ generated by $f$ and $g$ is primary to the maximal ideal of the local ring $A_{\mathfrak{m}}$. The intersection multiplicity is then defined simply to be the length of the quotient $A_{\mathfrak{m}} /(f, g)$, or, equivalently, its dimension over the field $k$.

There is more than one way to generalize this to an arbitrary dimension $d$. First, one can take the intersection of $d$ hyperplanes; in the case of affine space, for example, this can be done in the same way as curves in the plane. One can also define the intersection of two subvarieties (or subschemes). These subvarieties will be defined locally by ideals $I$ and $J$ at a point of intersection corresponding to a maximal ideal $\mathfrak{m}$. However, in this case, defining the intersection multiplicity to be the length of $A_{\mathfrak{m}} /(I, J)$ does not work; for example, Bézout's Theorem in projective space would not hold with this definition. What Serre did was to correct this definition by taking an Euler characteristic involving higher Tor modules. He defined the intersection multiplicity for any pair of modules $M$ and $N$ over a regular local ring $A$ such that $M \otimes N$ has finite length as follows.

$$
\chi(M, N)=\sum_{i=0}^{d}(-1)^{i} \text { length }\left(\operatorname{Tor}_{i}^{R}(M, N)\right) .
$$

The case of subvarieties above is where $M=A_{\mathfrak{m}} / I$ and $N=A_{\mathfrak{m}} / J$. In this case, letting $A_{\mathfrak{m}}=R$, we have $\operatorname{Tor}_{0}^{R}(M, N)=\operatorname{Tor}_{0}^{R}(R / I, R / J)=R / I \otimes_{R} R / J=$ $R /(I, J)$, so that the previous definition appears as the first term in this alternating sum. Serre's definition has many nice properties, such as additivity in each variable, but now some conditions which were clear before, such as the fact that it is nonnegative, are not so clear. Serre stated three conjectures which are equivalent to the four we give here. The notation $\mathrm{M}_{i}$ refers to Hochster's diagram.

Conjecture 1. (i) $\left(\mathrm{M}_{0}\right) \operatorname{dim}(M)+\operatorname{dim}(N) \leq \operatorname{dim}(R)$.

(ii) $\left(\mathrm{M}_{1}\right.$ : Vanishing) If $\operatorname{dim}(M)+\operatorname{dim}(N)<\operatorname{dim}(R)$, then $\chi(M, N)=0$.

(iii) (Nonnegativity) $\chi(M, N) \geq 0$.

(iv) $\left(\mathrm{M}_{2}\right.$ : Positivity) If $\operatorname{dim}(M)+\operatorname{dim}(N)=\operatorname{dim}(R)$, then $\chi(M, N)>0$.

Serre's original conjectures, as stated in Serre [62], V.B.3 and V.B.4, were nonnegativity, $\mathrm{M}_{0}$, and that $\operatorname{dim}(M)+\operatorname{dim}(N)=\operatorname{dim}(R)$ if and only if $\chi(M, N)>0$. The reason for stating them the way we did comes from later developments.

Before continuing, it will be good to go over some of the issues that arose in studying these questions, since they have been part of this subject ever since. First, there are three basic cases. Since $R$ is a regular local ring it is an integral domain and has a maximal ideal $\mathfrak{m}$ and residue field $k$. The cases are

(i) Characteristic zero: $R$ contains a field of characteristic zero.

(ii) Positive characteristic: $R$ contains a field of positive characteristic $p$ for some $p$.

(iii) Mixed characteristic: $R$ has characteristic zero but $k$ has positive characteristic $p$ for some $p$. 
The first two cases are called the equicharacteristic case. The mixed characteristic case can be further divided into the unramified case, in which the prime $p$ is not in $\mathfrak{m}^{2}$, and the ramified case, in which it is in $\mathrm{m}^{2}$. The most difficult case for these conjectures and many others is the ramified case in mixed characteristic.

The method of proof used by Serre in the equicharacteristic case, called "reduction to the diagonal", goes roughly as follows. First, one shows that the statments hold if one of the modules, say $M$, is of the form $R / I$, where $I$ is an ideal generated by a regular sequence (see Serre [62, Section IV.A.3]). If $R$ is a complete equicharacteristic regular local ring, the Cohen structure theorem says that it is a power series ring over a field. If we now have arbitrary modules $M$ and $N$ over a power series ring $k\left[\left[X_{1}, \ldots, X_{d}\right]\right]$, we think of $N$ as being a module over another power series ring $k\left[\left[Y_{1}, \ldots, Y_{d}\right]\right]$ and consider the "complete tensor product" $M \hat{\otimes}_{k} N$ as a module over $k\left[\left[X_{1}, \ldots, X_{d}, Y_{1}, \ldots, Y_{d}\right]\right]$; notice that the tensor product is taken over the subfield $k$. Let $I$ denote the ideal of $k\left[\left[X_{1}, \ldots, X_{d}, Y_{1}, \ldots, Y_{d}\right]\right]$ generated by $\left(X_{1}-Y_{1}, \ldots, X_{d}-Y_{d}\right)$; these elements form a regular sequence and define the diagonal subscheme in $\operatorname{Spec}\left(k\left[\left[X_{i}, Y_{i}\right]\right]\right)$. Then one shows that

$$
M \otimes_{R} N \cong\left(M \hat{\otimes}_{k} N\right) \otimes_{k\left[\left[X_{i}, Y_{i}\right]\right]}\left(k\left[\left[X_{i}, Y_{i}\right]\right] / I\right)
$$

and similarly for higher Tors. This reduces the question to one of the form a regular ring modulo a regular sequence, where it all works. Needless to say there are a lot of details omitted here; the complete story can be found in Serre [62].

In addition to the equicharacteristic case, Serre proved these results in the case of an unramified ring of mixed characteristic, and he proved the first statement for general regular local rings.

Serre also stated conjectures about partial Euler characteristics; that is, sums of the form

$$
\chi_{i}(M, N)=\sum_{j=i}^{d}(-1)^{j-i} \text { length }\left(\operatorname{Tor}_{i}(M, N)\right) .
$$

We note that this gives the alternating sum of lengths of Tor, starting now with the term $\operatorname{Tor}_{i}(M, N)$ with a positive sign instead of $\operatorname{Tor}_{0}(M, N)=M \otimes_{R} N$. Serre proved that in the equicharacteristic case, we have $\chi_{i}(M, N) \geq 0$ for all $i \geq 0$, and, in fact, if $\operatorname{Tor}_{i}(M, N) \neq 0$ and $i>0$, then $\chi_{i}(M, N)>0$. This implies in particular the following for equicharacteristic rings in the case where $M \otimes_{R} N$ has finite length. In fact, this was a result of Auslander [1] for all pairs of modules over unramified regular local rings, and it was conjectured to be true in general.

Conjecture 2 (The Rigidity of Tor (1)). Let $M$ and $N$ be finitely generated modules over a regular local ring. Then if $\operatorname{Tor}_{i}(M, N)=0$ for some $i>0$, then $\operatorname{Tor}_{j}(M, N)=$ 0 for all $j \geq i$.

The general case of Rigidity ( $R$ is still assumed regular) was proven by Lichtenbaum in [43]. He also extended Serre's results on partial Euler characteristics to the 
unramified case for $i \geq 2$ or when $M$ and $N$ are torsion-free; Hochster [26] completed the proof in the unramified case. The conjecture on partial Euler characteristics is still open for ramified regular local rings of mixed characteristic.

In the remainder of this section we discuss later developments on these conjectures. $R$ is always assumed to be a regular local ring.

\subsection{The Vanishing Conjecture}

The first of the multiplicity conjectures to be proven was the Vanishing Conjecture. This was proven independently in Roberts [51] (see also [53]) and by Gillet and Soulé in [18] (see also [19]). Both of the proofs involved new machinery in either Algebraic Geometry or $K$-theory. Before discussing these developments we put them into a more recent context.

Let $A$ be a local ring, and let $M$ be a module of finite projective dimension. Then $M$ has a finite free resolution

$$
0 \rightarrow F_{k} \rightarrow F_{k-1} \rightarrow \cdots \rightarrow F_{0} \rightarrow M \rightarrow 0 .
$$

It is often more convenient to replace $M$ with its resolution

$$
0 \rightarrow F_{k} \rightarrow F_{k-1} \rightarrow \cdots \rightarrow F_{0} \rightarrow 0 .
$$

This is a perfect complex, which means a bounded complex of finitely generated free modules. If $M$ and $N$ are both modules of finite projective dimension, and

$$
0 \rightarrow G_{t} \rightarrow G_{t-1} \rightarrow \cdots \rightarrow G_{0} \rightarrow N \rightarrow 0
$$

is a free resolution of $N$, then the tensor product of complexes $F_{\bullet} \otimes G_{\bullet}$ gives a complex with homology $\operatorname{Tor}_{i}(M, N)$. Since all modules over a regular local ring have finite projective dimension, this means that Serre's multiplicity conjectures can be formulated in terms of perfect complexes.

We now let $K_{0}(A)$ denote the $K$-group of perfect complexes over a local ring $A$. $K_{0}(A)$ is defined to be the free abelian group with generators isomorphism classes $\left[F_{\bullet}\right]$ of perfect complexes with relations given by

(i) $\left[F_{\bullet}\right]=\left[F_{\bullet}^{\prime}\right]+\left[F_{\bullet}^{\prime \prime}\right]$ if there is a short exact sequence of complexes

$$
0 \rightarrow F_{\bullet}^{\prime} \rightarrow F_{\bullet} \rightarrow F_{\bullet}^{\prime \prime} \rightarrow 0 .
$$

(ii) $\left[F_{\bullet}\right]=\left[G_{\bullet}\right]$ if there exists a map of complexes $F_{\bullet} \rightarrow G_{\bullet}$ that induces an isomorphism on homology modules.

A map of complexes that induces an isomorphism on homology modules is called a quasi-isomorphism.

In addition to its structure as an abelian group, $K_{0}(A)$ has a product defined by the tensor product of complexes, which we have already seen is related to intersection 
multiplicities. We define the support of a complex to be the union of the supports of its homology modules, or, equivalently, the set of prime ideals $\mathfrak{p}$ for which the localization of the complex at $\mathfrak{p}$ is not exact. If $F_{\bullet}$ is a perfect complex with support $W$ and $G_{\bullet}$ one with support $Z$, then it is not hard to show that the support of $F_{\bullet} \otimes G$ • is $W \cap Z$. Putting this together, we can see that $K_{0}(A)$ has a filtration by support and this filtration is compatible with the product structure.

As mentioned above, the Vanishing Conjecture for regular rings was proven around 1985; there were two independent proofs using different methods. However, in both cases the main idea was to replace the above filtration by a grading with good properties. Suppose that we could give $K_{0}(R)$, for a regular local ring $R$, a grading by codimension, so that we had $K_{0}(R)=\oplus_{i=0}^{d} G_{i}$, where $G_{i}$ gave the component representing elements with support of codimension $i$, and satisfying the condition that the intersection pairing mapped $G_{i} \times G_{j}$ to $G_{i+j} \oplus G_{i+j+1} \oplus \cdots$. Then if $M$ and $N$ were modules (or perfect complexes) with $\operatorname{dim} M+\operatorname{dim} N<\operatorname{dim} R$, they would be represented by sums of elements of $G_{i}$ and $G_{j}$ respectively with $i+j>d$, so the intersection product would be zero. This, roughly, is what each of the proofs did.

In the proof be Gillet and Soule the grading was given by eigenspaces of Adams operations on $K_{0}(R)$; see [19] for details.

In Roberts [51] the grading was given by a map to the rational Chow group, which we define briefly. For a Noetherian $\operatorname{ring} A$, we define the $i$ th graded piece of the rational Chow group, denoted $\mathrm{CH}_{i}(A)_{\mathbb{Q}}$, to be the $\mathbb{Q}$ vector space on generators $[\mathfrak{p}]$, where $\mathfrak{p}$ is a prime ideal such that the dimension of $A / \mathfrak{p}$ is $i$ modulo an equivalence relation called rational equivalence. Rational equivalence is defined by $\operatorname{setting} \operatorname{div}(\mathfrak{q}, x)$ to zero in $\mathrm{CH}_{i}(A)$, where $\mathfrak{q}$ is a prime ideal such that $A / \mathfrak{q}$ has dimension $i+1, x$ is an element of $A$ not in $\mathfrak{q}$, and, letting $B=A / \mathfrak{q}$,

$$
\operatorname{div}(\mathfrak{q}, x)=\sum_{\mathfrak{p}} \operatorname{length}\left(B_{\mathfrak{p}} / x B_{\mathfrak{p}}\right)[\mathfrak{p}]
$$

where the sum is over $\mathfrak{p}$ with $\operatorname{dim}(A / \mathfrak{p})=i$. There is then a map $\tau$ from $K_{0}(A)$ to operators on $\mathrm{CH}_{i}(A)_{\mathbb{Q}}$. If $A$ is a regular local ring, we can replace dimension by codimension and obtain a grading with the properties above. For details see Fulton [16] and Roberts [53] and [56].

These techniques allowed one to prove the Vanishing Conjecture also in the case in which $M$ and $N$ are modules of finite projective dimension over a complete intersection. We will discuss other generalizations in a later section.

\subsection{Gabber's Proof of the Nonnegativity Conjecture}

The third of Serre's conjectures, Nonnegativity, was proven by Gabber around 1996. Gabber never published the proof, but a brief summary appears in Berthelot [3], and more extensive versions can be found in Hochster [27] and Roberts [57]. Again there was a new ingredient; this time it was a theorem of de Jong on the existence of regular 
alterations [39]. We give here a special case of this theorem which applies to this problem.

Theorem 1 (A. J. de Jong). Let $A$ be a local integral domain which is essentially of $f$ nite type over a discrete valuation ring. Then there exists a scheme $X$ with a projective map $X \rightarrow \operatorname{Spec}(A)$ such that

(i) $X$ is an integral regular scheme (that is, all the local rings of $X$ are regular).

(ii) The field of rational functions $k(X)$ is a finite extension of the field of fractions of $A$.

There is some work involved in reducing to the case in which $A$ is essentially of finite type over a discrete valuation ring, and even in this case the proof is quite nontrivial. We mention briefly where de Jong's theorem is applied. It suffices to show that $\chi(A / \mathfrak{p}, A / \mathfrak{q}) \geq 0$ for prime ideals $\mathfrak{p}$ and $\mathfrak{q}$ such that $(\mathfrak{p}, \mathfrak{q})$ is primary to the maximal ideal. The theorem is applied to one of the quotients, say $A / p$. The machinery of intersection theory must be extended to perfect complexes on schemes and projective morphisms as well as over commutative rings. One curious feature of the proof is that at one point it is necessary to assume that the original local ring is ramified; it is easy to reduce to this case but unexpected that it would be useful. The proof also gives a new proof of the Vanishing Conjecture. We refer to the references above for descriptions of the proof.

\subsection{The Positivity Conjecture}

The positivity conjecture remains open. There have been several approaches to it, and we mention two.

One approach is based on the following. Let $M$ and $N$ be two modules over a regular local ring $R$ such that $\operatorname{dim} M+\operatorname{dim} N=\operatorname{dim} R$. If $M$ is Cohen-Macaulay, its minimal free resolution has length $\operatorname{dim} R-\operatorname{dim} M$, and if $N$ is also Cohen-Macaulay, then the condition on the length of the resolution of $M$ implies that $\operatorname{Tor}_{i}(M, N)=0$ for $i>0$. Thus $\chi(M, N)$ is the length of $M \otimes_{R} N$, which is clearly positive. Hence if we can reduce to the case in which $M$ and $N$ are Cohen-Macaulay, we are done.

A method for reducing to this case is, first, to reduce to the case where $M$ and $N$ are of the form $A / p$ by taking filtrations with quotients of this form; since the Vanishing Conjecture holds, we can reduce to modules of this form. If we could now find an $A / \mathfrak{p}$-module of the dimension of $A / \mathfrak{p}$ which was Cohen-Macaulay for any $\mathfrak{p}$, we could, again using vanishing, reduce to the case in which $M$ and $N$ are CohenMacaulay and complete the proof. The missing fact is the existence of what are called "small Cohen-Macauly modules"; these will be discussed in a later section. (What we have just described is the arrow from "Small C-M Modules" to " $\left(\mathrm{M}_{1} \Rightarrow \mathrm{M}_{2}\right)$, regular case" in Hochster's diagram.)

The other, more recent, attempts to prove the Positivity Conjecture use Gabber's construction. Kurano and Roberts [42] give a criterion for positivity to hold using this 
construction. Dutta [10] gives a formula for intersection multiplicities using the blowup of the maximal ideal of a regular local ring, again using Gabber's ideas. It is not clear whether any of these methods will lead to a proof of positivity, however, and that conjecture remains open.

\section{The Peskine-Szpiro Intersection Conjecture}

Serre's introduction of homological methods into intersection theory created much more interest in questions on homological algebra, and, in particular, properties of modules of finite projective dimension. The Auslander-Buchsbaum-Serre theorem states that every $R$-module has finite projective dimension if and only if the ring $R$ is regular, so one point of view is that properties of modules over regular local rings should extend to properties of modules of finite projective dimension over arbitrary local rings. One direction was to generalize the multiplicity properties themselves; this will be considered in the next section. A different direction was started by Peskine and Szpiro with their "Intersection Theorem". This was a main theorem of their paper Dimension projective finie et cohomologie locale, which was one of the most important papers in the development of the Homological Conjectures.

The Peskine-Szpiro Intersection Conjecture states:

Conjecture 3. Let $A$ be a local ring, let $M$ be an $A$-module of finite projective dimension, and let $N$ be a module such that $M \otimes N$ has finite length. Then the Krull dimension of $N$ is less than or equal to the projective dimension of $M$.

They stated this result as a theorem rather than a conjecture, since it was a theorem for rings of positive characteristic and rings essentially of finite type over a field of characteristic zero. We discuss this in more detail below.

In some ways this conjecture is analogous to Serre's conjectures. By the AuslanderBuchsbaum Theorem, the depth of a module is related to the projective dimension (if finite) by

$$
\text { projdim } M+\operatorname{depth} M=\operatorname{depth} A \text {. }
$$

or

$$
\operatorname{projdim} M=\operatorname{depth} A-\operatorname{depth} M \text {. }
$$

Thus the Peskine-Szpiro Theorem can be stated that

$$
\operatorname{dim} N+\operatorname{depth} M \leq \operatorname{depth} A \text {. }
$$

This is analogous, but certainly not equivalent, to the Serre theorem. Its interest lies in the fact that it implies several other conjectures from that time, of which we state two. 
Conjecture 4 (Bass). If a ring $A$ has a finitely generated nonzero module of finite injective dimension, then $A$ is Cohen-Macaulay.

Conjecture 5 (Auslander). Let $M$ be a finitely generated module of finite projective dimension. If $a \in A$ is a nonzerodivisor on $M$, then $a$ is a nonzerodivisor on $A$.

We refer to the paper of Peskine and Szpiro [47] for proofs that these conjectures are implied by the Intersection Conjecture.

A newer version of the Intersection Conjecture was introduced shortly thereafter; it is in the spirit of generalizing from modules to complexes referred to in the previous section.

Conjecture 6 (The New Intersection Conjecture). Let $A$ be a local ring of dimension $d$. If

$$
0 \rightarrow F_{k} \rightarrow F_{k-1} \rightarrow \cdots \rightarrow F_{0} \rightarrow 0
$$

is a complex of finitely generated free modules such that $H_{i}\left(F_{\bullet}\right)$ has finite length for each $i$ and $H_{0}\left(F_{\bullet}\right) \neq 0$, then $k \geq d$.

That this implies the original conjecture can be seen by applying the New Intersection Conjecture to a projective resolution of $M$ tensored with a suitable module of the form $A / \mathfrak{p}$ for $\mathfrak{p}$ a prime ideal of $A$ in the support of $N$.

In addition to stating this conjecture and several others, Peskine and Szpiro introduced two methods that are still very much in use in this area. Perhaps the most important is the use of the Frobenius map and reduction to positive characteristic. We briefly recall how this works.

Let $A$ be a ring of positive characteristic $p$. Then the Frobenius map, which we denote $F$, is the ring homomorphism defined by $F(a)=a^{p}$; it is a ring homomorphism since $p=0$ on $A$ so $(a+b)^{p}=a^{p}+b^{p}$ for all $a$ and $b$ in $A$. The basic idea of using this map to prove conjectures is to assume that there is a counterexample, and then to take a limit over powers of the Frobenius map to obtain a contradiction. A simpler method, which works sometimes, is to show that a high enough power of the Frobenius map produces an example that can be shown not to exist.

The second step in this process is to reduce the characteristic zero case to the case of positive characteristic. Peskine and Szpiro introduced this method for this kind of problem, and it was completed by Hochster.

The procedure is fairly complicated, but one step, reduction from finitely generated over a field to positive characteristic, goes something like this. Given a counterexample over a ring that is a finitely generated ring over a field of characteristic zero, one first, using the fact that there are only finitely many elements to consider, reduces to the case of a ring that is finitely generated over the rational numbers, and then one reduces further to an example over a ring finitely generated over the integers. Finally, one shows that for all but finitely many primes $p$, the reduction modulo $p$ and gives a 
counterexample in characteristic $p$. Peskine and Szpiro used this method to prove the Intersection Conjecture in the case of a local ring essentially of finite type over a field of characteristic zero, and they also used the Artin approximation Theorem to extend this to the case of a ring whose completion was the completion of a ring essentially of finite type over a field of characteristic zero. Shortly thereafter Hochster was able to extend this method to the general equicharacteristic case.

\subsection{Hochster's Metatheorem}

One of the main results of Hochster [24] was the following.

Theorem 2. Let $\xi$ be a system of polynomial equations in $d+q$ variables $X_{1}, \ldots, X_{d}$ and $Y_{1}, \ldots, Y_{q}$ over $\mathbb{Z}$, say

$$
\begin{gathered}
F_{1}\left(X_{1}, \ldots, X_{d}, Y_{1}, \ldots, Y_{q}\right)=0 \\
\vdots \\
F_{t}\left(X_{1}, \ldots, X_{d}, Y_{1}, \ldots, Y_{q}\right)=0 .
\end{gathered}
$$

Suppose that $\xi$ has a solution in a local ring $R$ which contains a field of characteristic zero such that $\operatorname{dim}(R)=d$ and the values $x_{1}, \ldots, x_{d}$ for $X_{1}, \ldots, X_{d}$ form $a$ system of parameters for $R$.

Then there exists a local ring $S$ containing a field of characteristic $p>0$ such that $\operatorname{dim}(S)=d$ and there is a solution of $\xi$ such that the values $x_{1}^{\prime}, \ldots, x_{d}^{\prime}$ for $X_{1}, \ldots, X_{d}$ form a system of parameters for $S$.

The proof of this theorem used Artin Approximation, and it finished the characteristic zero case of several of the conjectures, including the Intersection Conjecture and various others that we will discuss below.

The case of mixed characteristic was proven in Roberts [52]. Like the Serre vanishing theorem, this used the theory of local Chern characters. Another essential ingredient was a theorem relating Chern characters in positive characteristic to limits over the Frobenius map. Details of this and more can be found in Roberts [53].

The other main technique introduced in the paper of Peskine and Szpiro was local cohomology. As this is a topic that is still extremely important in this area, we will review some of the important points. For more complete introductions to the subject we refer to Brodman and Sharp [5] and Twenty-Four Hours of Local Cohomology [38].

Let $A$ be, as usual, a commutative Noetherian ring, and let $I$ be an ideal of $A$. For any $A$-module $M$, we define the submodule $\Gamma_{I}(M)$ to be the set of $m \in M$ that are annihilated by a power of $I$. It is easy to see that this is indeed a submodule of $M$ and that $\Gamma_{I}$ defines a left exact functor from the category of $A$-modules to itself. The functor is not right exact, however, and the right derived functors of $\Gamma_{I}$ applied to a module $M$, denoted $H_{I}^{i}(M)$, are the local cohomology modules of $M$ with support in $I$. 
The most important case as far as the Homological Conjectures are concerned is the case in which $A$ is local and $I$ is the maximal ideal $\mathrm{m}$. We note that if $I$ and $J$ are ideals with the same support, so that we have $J^{n} \subseteq I$ and $I^{m} \subseteq J$ for some $m$ and $n$, then it is clear that $\Gamma_{I}(M)=\Gamma_{J}(M)$ for all modules $M$, and thus the local cohomology modules with supports in $I$ and $J$ are the same. If $\mathfrak{m}$ is the maximal ideal of a local ring $A$ of dimension $d$, we can thus replace $\mathfrak{m}$ by an ideal $I$ generated by a system of parameters $\left(x_{1}, \ldots, x_{d}\right)$. Now given a set of generators for $I$, there are two standard methods for computing the local cohomology modules.

First, we let $C^{\bullet}$ denote the complex

$$
0 \rightarrow A \rightarrow \prod_{i} A_{x_{i}} \rightarrow \prod_{i<j} A_{x_{i} x_{j}} \rightarrow \cdots \rightarrow A_{x_{1} x_{2} \cdots x_{d}} \rightarrow 0,
$$

where the $A$ at the left has degree zero and the $A_{x_{1} x_{2} \cdots x_{d}}$ at the right has degree $d$. The maps are given by the inclusions with appropriate signs. Then it can be shown that

$$
H_{I}^{i}(M)=H^{i}\left(M \otimes_{A} C^{\bullet}\right)
$$

for any $A$-module $M$.

The second method is as a direct limit. For each $n$ we take the Koszul complex $K^{\bullet}\left(x_{1}^{n}, \ldots, x_{d}^{n}\right)$. For $m>n$ there is a map of complexes from $K^{\bullet}\left(x_{1}^{n}, \ldots, x_{d}^{n}\right)$ to $K^{\bullet}\left(x_{1}^{m}, \ldots x_{d}^{m}\right)$. The limit of these is, in fact the above complex and tensoring with $M$ again gives local cohomology.

For a ring of positive characteristic one can also define local cohomology as a limit over powers of the Frobenius map, and this was one of the methods introduced by Peskine and Szpiro. We will not discuss this further here, but we will return to the topic of local cohomology in later sections.

One of the facts that is used over and over in studying these conjectures is the following. If we assume that $A$ is a complete domain of dimension $d$, then there is an element $c \neq 0$ that annihilates the local cohomology modules $H_{\mathfrak{m}}^{i}(A)$ for $i<d$. This was proven in Roberts [50], where the element $c$ was taken to be in a product of annihilators of the cohomology of a dualizing complex, and in Hochster and Huneke [31], where $c$ was taken to be an element such that the localization $A_{c}=A[1 / c]$ is Cohen-Macaulay. Keeping in mind that the ring $A$ is Cohen-Macaulay if and only if the local cohomology modules $H_{\mathfrak{m}}^{i}$ are zero for $i<d$, it is not surprising that this fact is useful for approaching these conjectures in the non-Cohen-Macaulay case; this method works especially well when combined with the use of the Frobenius map.

To conclude this section we note that Avramov, Buchweitz, and Iyengar have formulated a generalization of the New Intersection Conjecture, called the "Class Inequality", to differential modules. A complex is a special case of a differential module; the differential module is the direct sum of the modules in the complex with differential given by the sum of the boundary maps. They prove this inequality in the equicharacteristic case; the case of mixed characteristic is still open. We refer to [2] for details. 


\section{Generalizations of the Multiplicity Conjectures}

As has already been mentioned, one direction of research on these conjectures was to generalize Serre's conjectures to nonregular rings. We recall that we had defined the intersection multiplicity $\chi(M, N)$, where $M$ and $N$ are two finitely generated modules over a regular local ring $R$ with $M \otimes_{R} N$ of finite length to be

$$
\left.\chi(M, N)=\sum_{i=0}^{d}(-1)^{i} \text { length } \operatorname{Tor}_{i}(M, N)\right) .
$$

As stated, this would be defined over any local ring; however, the fact that $R$ is regular implies that higher Tors are zero, which, using the long exact sequence of Tors, implies that $\chi(M, N)$ is additive in $M$ and in $N$. Over a nonregular ring we need an extra condition, and the weakest condition which makes this work is that one of the modules, say $M$, has finite projective dimension; its projective dimension will still be at most $d$. We now restate the conjectures with this assumption.

Conjecture 7. Let $A$ be a local ring, and let $M$ and $N$ be finitely generated modules such that $M$ has finite projective dimension and $M \otimes_{A} N$ has finite length. Then

(i) $\left(\mathrm{M}_{0}\right) \operatorname{dim}(M)+\operatorname{dim}(N) \leq \operatorname{dim}(A)$.

(ii) $\left(\mathrm{M}_{1}\right.$ : Vanishing) If $\operatorname{dim}(M)+\operatorname{dim}(N)<\operatorname{dim}(A)$, then $\chi(M, N)=0$.

(iii) $\left(\mathrm{M}_{2}\right.$ : Positivity) If $\operatorname{dim}(M)+\operatorname{dim}(N)=\operatorname{dim}(A)$, then $\chi(M, N)>0$.

It is a rather remarkable fact that the first of these conjectures, which appears to be the most basic, is still open in this generality. It holds for many examples of modules of finite projective dimension, and there are many easy counterexamples if neither module has finite projective dimension, but it is not known in the case stated here, in spite of the fact that it is a rather simple statement about the nature of the support of a module of finite projective dimension.

\subsection{The Graded Case}

One of the remarkable results of Peskine and Szpiro was a Comptes Rendus article [48] in which they proved some of the conjectures for the graded case. More precisely, they assumed that $A$ is a standard graded ring over an Artinian local ring (such as a field), $M$ is a graded module of finite projective dimension, and $N$ is another graded module. In this case, $M$ has a finite free resolution by modules that are direct sums of $A\left(n_{i j}\right)$, the graded module $A$ with grading shifted by $n_{i j}$, for various $n_{i j}$. They gave a formula which allows one to compute the intersection multiplicities in terms of the $n_{i j}$ in such a way that they could prove all three parts of this conjecture.

In addition, they proved the following conjecture in the graded case:

Conjecture 8. Let $M$ be an $A$-module of finite projective dimension. Then

$$
\operatorname{grade}(M)=\operatorname{dim}(A)-\operatorname{dim}(M) .
$$


We recall that the grade of a module is the longest possible length of a regular sequence contained in the annihilator of $M$. This is the Codimension Conjecture (13) of Hochster's 1975 diagram; the word codimension was once used for what we now call grade. This conjecture is a statement about the prime ideals in the support of a module of finite projective dimension and holds, for example, for an equidimensional ring, but it is still open in general. For a discussion of this conjecture we refer to [55].

We make one final remark about the methods of this paper on the graded case. The authors said at the time that their method of computing intersection multiplicities through numerical invariants was a kind of "Riemann-Roch Theorem". This was in fact one of the main inspirations for later work on finding a Riemann-Roch Theorem in general. On the other hand, the question of whether this is really a version of the Riemann-Roch Theorem of Hirzebruch was not raised until later, and a direct proof that they agree was only given recently (see [58]).

We now return to the main topic of the Strong Multiplicity Conjectures.

As mentioned above, the first of these conjectures is still open. The second two, however, are false. This was an example of Dutta, Hochster, and McLaughlin [11] which was one of the turning points in research in this area. We present an outline of this example, leaving out the details.

Let $k$ be a field, and let $A$ be $k[X, Y, Z, Y] /(X Y-Z W)$ localized at the maximal ideal $(X, Y, Z, W)$ (or $k[[X, Y, Z, W]] /(X Y-Z W)$ if you prefer). Let $N=$ $A /(X, Z)$. We note that since $(X, Z)$ contains $X Y-Z W, N$ has dimension 2. The problem is to construct a module of finite length and finite projective dimension such that $\chi(M, N) \neq 0$. This is carried out by a detailed computation of a set of matrices representing the action of $X, Y, Z$, and $W$ on a finite dimensional vector space; the authors determine the precise conditions these matrices must satisfy and produce a set of large matrices satisfying them.

This counterexample also had influence on the theory of local Chern characters, showing that they did not vanish where predicted. More on this approach to the question can be found in Szpiro [65] and Roberts [53] and [56].

We mention a result of Sather-Wagstaff [61] which is similar to statement $\mathrm{M}_{0}$ above but where the hypothesis of finite projective dimension is replaced by a condition on multiplicity.

Theorem 3. Let $A$ be an excellent quasi-unmixed Cohen-Macaulay local ring that contains a field. Let $\mathfrak{p}$ and $\mathfrak{q}$ be prime ideals such that $A / \mathfrak{p} \otimes_{A} A / \mathfrak{q}$ has finite length and the multiplicity of $A_{\mathfrak{p}}$ is equal to the multiplicity of $A$. Then $\operatorname{dim}(A / \mathfrak{p})+\operatorname{dim}(A / \mathfrak{q}) \leq$ $\operatorname{dim}(A)$.

The interesting point here is that the condition on multiplicities is automatic for regular local rings, since the localization of a regular local ring is regular so both multiplicities are one, like the condition in $M_{0}$ that $M$ have finite projective dimension.

We now return to a discussion of further developments on counterexamples to this generalization of the Vanishing Conjecture. As mentioned above, the example of 
Dutta, Hochster, and McLaughlin had implications for local Chern characters. Let $A$ be a local domain of dimension $d$ which is either complete or essentially of finite type over a field (this is sufficient so that Chow groups and local Chern characters are defined). Associated to $A$ are two elements of the Chow group of $\mathrm{CH}_{*}(A)$. The first is the class $[A]$; since $A$ is a domain, 0 is a prime ideal and this defines an element of $\mathrm{CH}_{d}(A)$. The second is the local Todd class, denote $\tau(A)$, which is equal to $[A]$ up to elements of lower dimension, and which is what is used in formulas for multiplicities. If $A$ is a complete intersection, it can be shown that $\tau(A)=[A]$; there are no lower terms. The counterexample to vanishing enables one to construct an example of a Cohen-Macaulay domain $A$ of dimension 3 for which the dimension 2 component of $\tau(A)$, denoted $\tau_{2}(A)$, is not zero and (more important), there is a module of finite length and finite projective dimension whose local Chern character does not vanish on $\tau_{2}(A)$. This left open the question of whether there was a similar example where $A$ is Gorenstein. If $A$ is Gorenstein of dimension $d$, then it can be shown that $\tau_{d-1}(A)=0$, so any nonvanishing component would have to be of higher codimension.

First Kurano [41] provided an example of a Gorenstein ring of dimension 5 for which $\tau_{3}(A) \neq 0$. C. Miller and Singh [46] then gave an example, also Gorenstein of dimension 5, for which there exists a module of finite length and finite projective dimension whose local Chern character does not vanish on $\tau_{3}(A)$. In Roberts and Srinivas [60], a general theorem was proven for local rings $A$ which are localizations at the maximal ideal of a standard graded ring such that the associated projective scheme $X$ is smooth (this includes all the above examples). In the nice case in which the Chow group of $X$ is essentially the same as the cohomology of $X$ (which is also true in the above examples), the main theorem states that if $\eta$ is any cohomology class that is zero when intersected with the hyperplane section, intersection with $\eta$ can be represented by a module of finite length and finite projective dimension. This implies in particular that there is such a module for Kurano's example. It also means that counterexamples of this sort are quite natural when seen from the point of view of intersection theory in Algebraic Geometry.

In all of the discussion in this section, we have only assumed that one of the modules $M$ and $N$ has finite projective dimension. If we assume that both modules have finite projective dimension, the conjectures are still open. If the ring is a complete intersection, then the Vanishing Conjecture is known in this case. There is an example in Roberts [54] of two perfect complexes which define positive cycles for which the intersection multiplicity is negative (this cannot happen over regular local rings), which may suggest that the Positivity Conjecture does not hold in this generality.

However, there are no indications that the Vanishing Conjecture for two modules of finite projective dimension is not true, and this is one of the main open questions in this area at the present time. In the counterexamples described above, the module $M$ of finite projective dimension has finite length and the module $N$ has dimension less than the dimension of the ring. If $N$ also has finite projective dimension, the fact that its dimension is less than that of the ring implies that the alternating sum of ranks of 
modules in its resolution is zero, and it follows that $\chi(M, N)=0$. Thus one must look elsewhere if one hopes to find a counterexample to vanishing with both modules of finite projective dimension.

\subsection{The Generalized Rigidity Conjecture}

The conjecture on the Rigidity of Tor was also generalized from the regular case to the case in which one module had finite projective dimension. This was disproven by Heitmann in [20].

It could be thought that with the regular case proven and the generalized case false, that would be the end of the story for the question of Rigidity of Tor. However, there have been several further results in this area, particularly for modules over hypersurfaces. We give two examples.

First, we have the following theorem due to Huneke and R. Wiegand [37] (their actual theorem is a little stronger than this).

Theorem 4. Let $A=R /(f)$ be a hypersurface of dimension $d$, where $R$ is an unramified regular local ring of dimension $d+1$. Let $M$ and $N$ be A-modules such that

(i) $M \otimes_{A} N$ has finite length.

(ii) $\operatorname{dim}(M)+\operatorname{dim}(N) \leq d$.

Then if $\operatorname{Tor}_{i}(M, N)=0$ for some $i \geq 0$, then $\operatorname{Tor}_{j}(M, N)=0$ for $j \geq i$.

A more recent result on this topic is due to Hailong Dao [6]. This uses a construction of Hochster for hypersurfaces which had been introduced earlier to study these conjectures. Let $A=R /(f)$ be a hypersurface, and suppose also that $A$ is an isolated singuarity. Then a resolution of a finitely generate module is eventually periodic of period 2 by results of Eisenbud [12], and the $\operatorname{Tor}_{i}(M, N)$ are eventually of finite length since $A$ has an isolated singularity. Hochster defined

$$
\theta(M, N)=\text { length }\left(\operatorname{Tor}_{2 i}(M, N)\right)-\operatorname{length}\left(\operatorname{Tor}_{2 i+1}(M, N)\right) .
$$

Dao proved the following theorem. Here $A$ is a hypersurface of the form $R /(f)$, but in addition to $R$ being regular, it must also be a power series ring over a field or a discrete valuation ring, so that in particular all of Serre's multiplicity conjectures hold.

Theorem 5. Let $A$ be as above, and let $M$ and $N$ be two finitely generated A-modules. Assume that $\theta(M, N)=0$. If $\operatorname{Tor}_{i}(M, N)=0$ for some $i \geq 0$, then $\operatorname{Tor}_{j}(M, N)=0$ for $j \geq i$.

We also want to mention an example of Dutta [9], which shows that the partial Euler characteristic $\chi_{2}(M, N)$ can be negative for two modules of finite projective dimension over a Gorenstein ring. While the original counterexample to vanishing 
shows that Serre's conjectures on partial Euler characteristics cannot be extended in general, Dutta's example is interesting in that it shows that they can fail even in a case where vanishing holds.

\section{The Monomial, Direct Summand, and Canonical Element Conjectures}

The first two of these conjectures, the Monomial and Direct Summand Conjectures, were introduced by Hochster and are listed in his diagram as consequences of the existence of big Cohen-Macaulay modules (which we will discuss below). They can all be proven in the equicharacteristic case by reduction to positive characteristic as outlined in the previous section.

The Direct Summand Conjecture states:

Conjecture 9 (Direct Summand Conjecture). If $R$ is a regular local ring and $S$ is a module-finite extension of $R$, then $R$ is a direct summand of $S$ as an $R$-module.

The Monomial Conjecture states:

Conjecture 10 (Monomial Conjecture). If $x_{1}, \ldots, x_{d}$ is a system of parameters for a local ring $R$, then

$$
x_{1}^{t} x_{2}^{t} \cdots x_{d}^{t} \notin\left(x_{1}^{t+1}, \ldots, x_{d}^{t+1}\right) .
$$

It is not too difficult to show that these two conjectures are equivalent. Shortly thereafter Hochster formulated the Canonical Element Conjecture. There are several versions of this conjecture, and we state three. The first shows why it is called the "Canonical Element" conjecture, and the second and third are easier to compute. In all three statements we let $A$ be a local ring of dimension $d$ with maximal ideal $\mathfrak{m}$ and residue field $k$.

Conjecture 11. Canonical Element Conjecture I: Let syz ${ }^{d}(k)$ be the $d$ th syzygy module of $k$, so that there is an exact sequence

$$
0 \rightarrow \operatorname{syz}^{d}(k) \rightarrow F_{d-1} \rightarrow \cdots \rightarrow F_{0} \rightarrow k \rightarrow 0
$$

where the $F_{i}$ are free modules. Using the Yoneda definition of Ext, this $d$-fold extension defines an element of $\operatorname{Ext}^{d}\left(k, \operatorname{syz}^{d}(k)\right)$, and hence, mapping to the limit, an element $\eta$ of

$$
\lim _{\vec{n}} \operatorname{Ext}^{d}\left(A / \mathfrak{m}^{n}, \operatorname{syz}^{d}(k)\right)=H_{\mathfrak{m}}^{d}\left(\operatorname{syz}^{d}(k)\right) .
$$

Then $\eta \neq 0$. ( $\eta$ is the "canonical element"). 
Canonical Element Conjecture II: Let $x_{1}, \ldots, x_{d}$ be a system of parameters for $A$. Let $K_{*}$ be the Koszul complex on $x_{1}, \ldots, x_{d}$ and let $F_{*}$ be a free resolution of $k$. Suppose we have

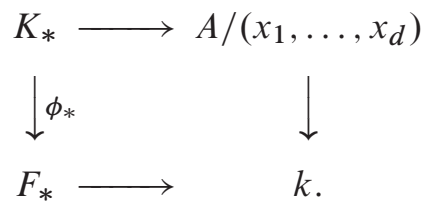

Then $\phi_{d} \neq 0$.

Canonical Element Conjecture III: Let $x_{1}, \ldots, x_{d}$ be a system of parameters for $A$. Let $K_{*}$ be the Koszul complex on $x_{1}, \ldots, x_{d}$ and let $F_{*}$ be a free resolution of $A /\left(x_{1}, \ldots, x_{d}\right)$. Suppose we have

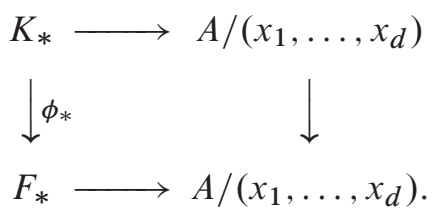

Then the image of $\phi_{d}$ is not contained in $\mathfrak{m} F_{d}$.

These three are not obviously equivalent, and proofs of their equivalence and the fact that they are also equivalent to the Direct Summand and Monomial Conjectures can be found in Hochster [25] and Dutta [7]. It should be pointed out that the fact that the Monomial Conjecture or Direct Summand Conjecture implies the Canonical Element Conjecture is quite nontrivial for rings of positive or mixed characteristic; in characteristic zero the Direct Summand Conjecture is trivial and holds for any normal domain since the trace map can be divided by the degree of the extension of quotient fields. It is equivalent to the Canonical Element Conjecture in characteristic zero only in the sense that both are known to be true.

These three conjectures have been among the most seriously studied during the years since their formulation. They follow from the existence of big Cohen-Macaulay modules, and they can be proven directly by the method of reduction to positive characteristic outlined above in the equicharacteristic case. Since Cohen-Macaulay modules exist in dimension at most two, the conjectures have been known since the beginning in any characteristic in dimension less than three. The reference Hochster [25] also contains many more interesting results on this topic, and it includes the fact that to prove the direct summand conjecture it suffices to prove it in the case in which $R$ is an unramified regular local ring, a condition that is often assumed in studying the problem.

Although the canonical element of the conjecture looks somewhat mysterious, there are a number of conjectures similar to the ones we are discussing that involve the 
canonical module or, more generally, the dualizing complex of a local ring. For examples, we refer to Strooker and Stückrad [64] and Dutta [8], where the Monomial Conjecture is related to properties of a dualizing module.

A major breakthrough on these conjectures came in 2003, when Heitmann [22] proved the Direct Summand Conjecture (and therefore several others) in dimension three in mixed characteristic. This proof did not involve new machinery, but rather it showed, by prodigious computations, that if one had a non-Cohen-Macaulay ring of mixed characteristic of dimension three for which $p, x, y$ is a system of parameters, and if we have a relation $a p^{N} \in(x, y)$, then for any integer $n$, in some finite extension we have that $a p^{1 / n} \in(x, y)$. Thus we do not get $a \in(x, y)$ (which would of course be true in a Cohen-Macaulay ring), but something close, and Heitmann proved that this is enough to prove the Direct Summand Conjecture. A little later Heitmann [23] showed that the system of parameters $p, x, y$ can be replaced by any system of parameters; it is not necessary to assume that one of them is $p$. We will discuss this further below.

\section{Cohen-Macaulay Modules and Algebras}

The importance of finding Cohen-Macaulay modules was clear from the beginnings of this subject. Serre had already shown, as we mentioned above, that if $M$ and $N$ are Cohen-Macaulay in the situation of his positivity conjecture, then $\operatorname{Tor}_{i}(M, N)=0$ for all $i>0$, so that $\chi(M, N)$ is simply the length of $M \otimes N$, which is clearly positive. It is also not difficult to show that most of the conjectures we have discussed over a ring $A$ of dimension $d$ will follow if there exists a finitely generated Cohen-Macaulay module of dimension $d$. Such a module is called a "small Cohen-Macaulay module" (it is also sometimes called a "maximal Cohen-Macaulay module", which admittedly is not terribly consistent terminology).

There are rings which cannot have small Cohen-Macaulay modules, such as noncatenary rings, but these can be considered pathological. In addition, most of the conjectures we have been discussing can be reduced to the complete case, and it would suffice to show that small Cohen-Macaulay modules exist for complete domains.

Conjecture 12. Every complete local domain has a small Cohen-Macaulay module.

This conjecture is easy if the dimension of $A$ is at most two, since in dimension one any domain is Cohen-Macaulay, and in dimension two one can take the normalization, which is Cohen-Macaulay. However, very little is known beyond that case. There is an example for graded rings attributed independently to Peskine and Szpiro, Hartshorne, and Hochster; they showed that small Cohen-Macaulay modules exist for graded domains of positive characteristic in dimension three (for a proof see Hochster [29]). Dan Katz [40] proved that there is such a module for extensions obtained by adjoining a $p$ th root to an unramified regular local ring. On the other hand, there are 
non-Cohen-Macaulay unique factorization domains, which cannot have small CohenMacaulay modules of rank one [4]. But basically this question is completely open.

One of the new developments in Hochster's 1975 paper was to introduce a weaker version of Cohen-Macaulay modules, called "big" Cohen-Macaulay modules. Their existence does not imply the implication in Serre's conjecture, but it does imply the Intersection Conjecture, the Canonical Element Conjecture, and several others.

Let $A$ be a local ring with system of parameters $x_{1}, \ldots, x_{d}$. A big Cohen-Macaulay module is an $A$-module $M$ such that

(i) $x_{1}, \ldots, x_{d}$ form a regular sequence on $M$.

(ii) $M /\left(x_{1}, \ldots, x_{d}\right) M \neq 0$.

The second condition is crucial; there are numerous infinitely generated modules that satisfy the first condition but not the second, and without this condition none of the stated implications hold. If $M$ is a small Cohen-Macaulay module, however, Nakayama's Lemma implies that condition 2 holds.

Conjecture 13. Every local ring has a big Cohen-Macaulay module.

Like the conjectures in the previous section, this conjecture is known in the equicharacteristic case and for rings of dimension at most 3. The basic method used by Hochster in [24] was to kill any bad relations as follows. If $M$ is not Cohen-Macaulay, there exists an $m \in M$ such that $x_{i} m \in\left(x_{1}, \ldots, x_{i-1}\right)$ but $m \notin\left(x_{1}, \ldots, x_{i-1}\right)$ for some $i$. We then extend $M$ to $M^{\prime}=M \oplus A^{i-1}$ modulo the relation $\left(m, x_{1}, \ldots, x_{i-1}\right)$; this puts the image of $m$ into the submodule $\left(x_{1}, \ldots, x_{i-1}\right) M^{\prime}$. We then take a huge and carefully constructed limit, and it is then easy to see that the limit will satisfy the first condition. The problem is to show that the second condition also holds. The original proof in the equicharacteristic case involves the Frobenius map and Hochster's Metatheorem. The proof in dimension 3 uses Heitmann's results.

\subsection{Weakly Functorial Big Cohen-Macaulay Algebras}

A further development in this area was the introduction of big Cohen-Macaulay algebras. One method for construction such an algebra is similar to that of big CohenMacaulay modules mentioned earlier, but instead of taking a free module in the extension and dividing by the relation as above, one takes a free commutative algebra; that is, a polynomial ring and again divides by an appropriate relation and takes a limit. For the applications one would like it to be functorial; this does not seem possible, but when they exist they can be made "weakly functorial", which is enough for many applications. We give the definitions.

Let $R$ be a local ring with system of parameters $x_{1}, \ldots, x_{d}$. A big Cohen-Macaulay algebra is an algebra $A$ over $R$ such that

(i) $x_{1}, \ldots, x_{d}$ form a regular sequence on $A$.

(ii) $A /\left(x_{1}, \ldots, x_{d}\right) A \neq 0$. 
"Weakly Functorial" means that given $R \rightarrow S$, one can find Cohen-Macaulay algebras $A$ and $B$ and a diagram

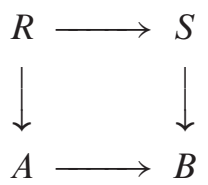

Conjecture 14. Every local ring has a big Cohen-Macaulay algebra, and for any map of local rings they can be chosen to be weakly functorial in the sense given above.

It can be seen that this is a considerably stronger conjecture than the existence of big Cohen-Macaulay modules, which in turn is stronger than the conjectures of the previous section. However, it has so far been the case that once methods had been developed to prove one of these conjectures, it can be applied to prove the existence of weakly functorial big Cohen-Macaulay algebras. An example is the case of dimension 3 in mixed characteristic, where the results of Heitmann's proof of the Direct Summand conjecture were used by Hochster to prove this conjecture as well [28].

A remarkable theorem appeared in 1990, with the proof by Hochster and Huneke that $R^{+}$is Cohen-Macaulay for $R$ a domain of positive characteristic [33]. Here $R^{+}$ is the absolute integral closure of $R$, which means the integral closure in the algebraic closure of its quotient field. This was later given a much simpler proof by Huneke and Lyubeznik [36]. This is better than just the existence, since it gives a specific construction in the positive characteristic case.

The existence of weakly functorial big Cohen-Macaulay algebras has many applications; for example, they imply the conjectures on the vanishing of maps of Tor and that direct summands of regular local rings are Cohen-Macaulay that we will state below. For more details on the existence and applications of such algebras we refer to Hochster and Huneke [34].

\section{The Syzygy Conjecture and the Improved New Intersection Conjecture}

Evans and Griffiths proved the following theorem for rings containing a field [13].

Theorem 6. Let $A$ be a Cohen-Macaulay local ring containing a field, and let $M$ be a finitely generated $k$ th module of syzygies that has finite projective dimension. If $M$ is not free, then $M$ has rank at least $k$.

In proving this conjecture it turned out that a stronger version of the Intersection Conjecture was one of the key points in the proof. This was named the "Improved New Intersection Conjecture". 
Conjecture 15. Let $A$ be a local ring of dimension $d$, and let

$$
0 \rightarrow F_{k} \rightarrow \cdots \rightarrow F_{1} \rightarrow F_{0} \rightarrow 0
$$

be a complex of finitely generated free modules such that $H_{i}\left(F_{\bullet}\right)$ has finite length for $i \geq 1$ and the cokernel of $F_{1} \rightarrow F_{0}$ has a minimal generator annihilated by a power of the maximal ideal. Then $k \geq d$.

The original New Intersection Conjecture is the case where the cokernel of $F_{1} \rightarrow$ $F_{0}$ is itself of finite length (and nonzero). While this is a version of the Intersection Conjecture, it is in fact stronger, and is equivalent to the Canonical Element Conjecture and the others in that group. Thus it is now known in the equicharacteristic case and in dimension at most 3 .

Recently Evans and Griffith have proven their Syzygy Theorem for certain graded modules of mixed characteristic [15]. They also have a more extensive account of problems concerning syzygies in [14].

\section{Tight Closure Theory}

In 1985 Hochster and Huneke introduced the concept of tight closure. It is defined for equicharacteristic rings; to keep the discussion simple we will give the definition for integral domains of positive characteristic.

Definition 1. Let $I$ be an ideal of an integral domain $A$ of positive characteristic $p$. The tight closure of $I$, denoted $I^{*}$, is the set of $a \in A$ for which there is an element $c \neq 0$ in $A$ such that $c a^{p^{e}} \in I^{\left[p^{e}\right]}$ for all $e \geq 0$.

Here $I^{\left[p^{e}\right]}$ is the ideal generated by $i^{p^{e}}$ for all $i \in I$. Tight closure is also defined for rings of characteristic zero using a method of reduction to positive characteristic. We refer to Huneke's notes from the Fargo conference [35] and its bibliography for much more information about tight closure. We will mention some connections to the problems we have been discussing here.

First of all, tight closure made it possible to give nicer proofs of some of the Homological Conjectures, such as the Monomial Conjecture and the existence of big Cohen-Macaulay modules, in the equicharacteristic case, although the basic idea, reduction to positive characteristic and the use of the Frobenius map, was similar to methods used earlier. One of the first ideas that arose from this was to attempt to find a similar closure operation that would work in mixed characteristic. A list of the desired properties of such a closure operation can be found, for example, in the Introduction of the notes of Huneke cited above; for the purposes of these conjectures, one of the main ones is "colon-capturing", which states that if $x_{1}, \ldots, x_{d}$ is a system of parameters, and if $a x_{i} \in\left(x_{1}, \ldots, x_{i-1}\right)$ for some $i$, then $a$ is in the closure of $\left(x_{1}, \ldots, x_{i-1}\right)$. A closure operation with all the right properties has not been found; however, this 
did inspire some new methods; in particular, Heitmann's proof of the Direct Summand Conjecture in dimension three was motivated in part by an attempt to show that "full extended plus closure" satisfies the colon-capturing condition in mixed characteristic. In this part of the discussion we assume that $A$ is a complete local domain and recall that $A^{+}$is the integral closure of $A$ in the algebraic closure of its quotient field.

Definition 2. If $x \in A$, then $x$ is in the full extended plus closure of $I$ if there exists $c \neq 0 \in A$ such that for every positive integer $n, c^{1 / n} x \in\left(I, p^{n}\right) A^{+}$. We write $x \in I^{\mathrm{epf}}$.

A similar closure operation had been defined by Hochster and Huneke in [32]. They defined the "dagger closure" to as follows (with the same assumptions on $A$ ). In this definition we fix a valuation $v$ on $A^{+}$with values in $\mathbb{Q} \cup\{\infty\}$ which is nonnegative on $A^{+}$and positive on the maximal ideal of $A^{+}$.

Definition 3. If $x \in A$, then $x$ is in the dagger closure of $I$ if there exist elements $u \in A^{+}$of arbitrarily small positive order with $u x \in I A^{+}$. We write $x \in I^{\dagger}$.

It is easy to see that $I^{\text {epf }} \subseteq I^{\dagger}$. The main result of Hochster and Huneke was that dagger closure and tight closure are the same in positive characteristic, so it made sense to try to show that dagger closure satisfies the colon-capturing property. Heitmann's results show that this is true in dimension three.

In addition to leading to these developments on the original homological conjectures, the connections that tight closure demonstrated with other areas inspired some new conjectures.

Conjecture 16 (Vanishing of Maps of Tors). Let $R$ be a regular ring, $A$ a module finite torsion-free extension of $R$, and $T$ a regular local ring with a map $\phi$ from $A$ to $T$. Then for every $R$-module $M$ and every $i \geq 1$, the map induced by $\phi$ from $\operatorname{Tor}_{i}^{R}(M, A)$ to $\operatorname{Tor}_{i}^{R}(M, T)$ is zero.

This conjecture has a similar flavor to some of the previous ones, particularly in the case where $T$ is a finite $A$-module, and it implies several of them. However, this one is much more general; $T$ could be an infinite extension, or on the other hand it could be the residue field of $A$ if $A$ is a local ring. It is known in the equicharacteristic case. We refer to Hochster [30] for a more complete discussion of this conjecture and its relation to other ones.

Another result of tight closure was to give a simple proof in characteristic zero that invariants of certain group actions on regular rings are Cohen-Macaulay. They proved, in fact, that a direct summand of a regular ring in equal characteristic is CohenMacaulay; it is a conjecture in mixed characteristic. 
Conjecture 17. A direct summand of a regular ring is Cohen-Macaulay.

If we apply this to the $R$-module $M=R /\left(x_{1}^{t+1}, \ldots, x_{d}^{t+1}, x_{1}^{t} x_{2}^{t} \cdots x_{d}^{t}\right)$ where $R$ is (regular) local of dimension $d$ and $x_{1}, \ldots, x_{d}$ is a system of parameters, it is not hard to see that this conjecture implies the Monomial Conjecture and hence also the Direct Summand Conjecture. In fact, it is equivalent to a stronger version of this conjecture.

\section{The Strong Direct Summand Conjecture}

In this section we discuss several recent variations on conjectures related to Direct Summands.

Conjecture 18 (Strong Direct Summand Conjecture). Let $R$ be a regular local ring and let $A$ be a finite extension of $R$. Let $Q$ be a height one prime ideal of $A$ containing $x R$, where $x$ is a minimal generator of the maximal ideal of $R$. Then $x R$ is a direct summand of $Q$.

At first sight this appears to be a rather gratuitous generalization of the Direct Summand Conjecture. It is indeed a generalization, since if this holds, then since $x A$ is contained in $Q$, the splitting map from $Q$ to $x R$ induces one from $x A$ to $x R$, and dividing by $x$ we obtain one from $A$ to $R$. Its importance comes from the surprising fact that it is equivalent to the Vanishing Conjecture for maps of Tors. This was proven by N. Ranganathan in [49].

She also had a strong version of the Monomial Conjecture:

Conjecture 19 (Strong Monomial Conjecture). Let $A$ be a local domain with system of parameters $\left(x_{1}, \ldots, x_{d}\right)$. Let $Q$ be a height one prime of $A$ containing $x_{1}$. Then

$$
x_{1}\left(x_{1} x_{2} \cdots x_{d}\right)^{t} \notin\left(x_{1}^{t+1}, \ldots, x_{d}^{t+1}\right) Q
$$

for all $t>0$.

A much more complete discussion of the conjectures of the last two sections and relations between them can be found in Hochster [29] and [30].

Recent work on the Strong Monomial Conjecture can be found in McCullough [45].

We include here the updated version of Hochster's diagram from the 2004 Minicourse at the University of Utah. For the most part, the conjectures in the new diagram have been stated since the earlier diagram; the main exceptions are some of those in the lower right. Even here some of the implications are new (since 1975). Also, the Intersection and New Intersection conjectures have actually been proven, but they are included here to show how they fit with some of the more recent conjectures. 


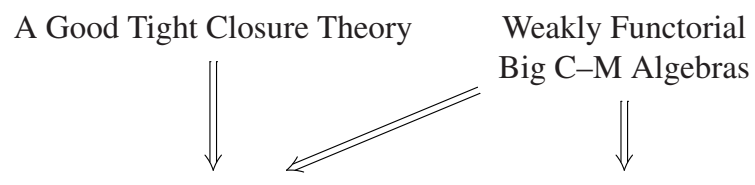

Strong Direct $\Longleftrightarrow$ Vanishing for Maps of Tor $\quad$ Big C-M Algebras

Summand
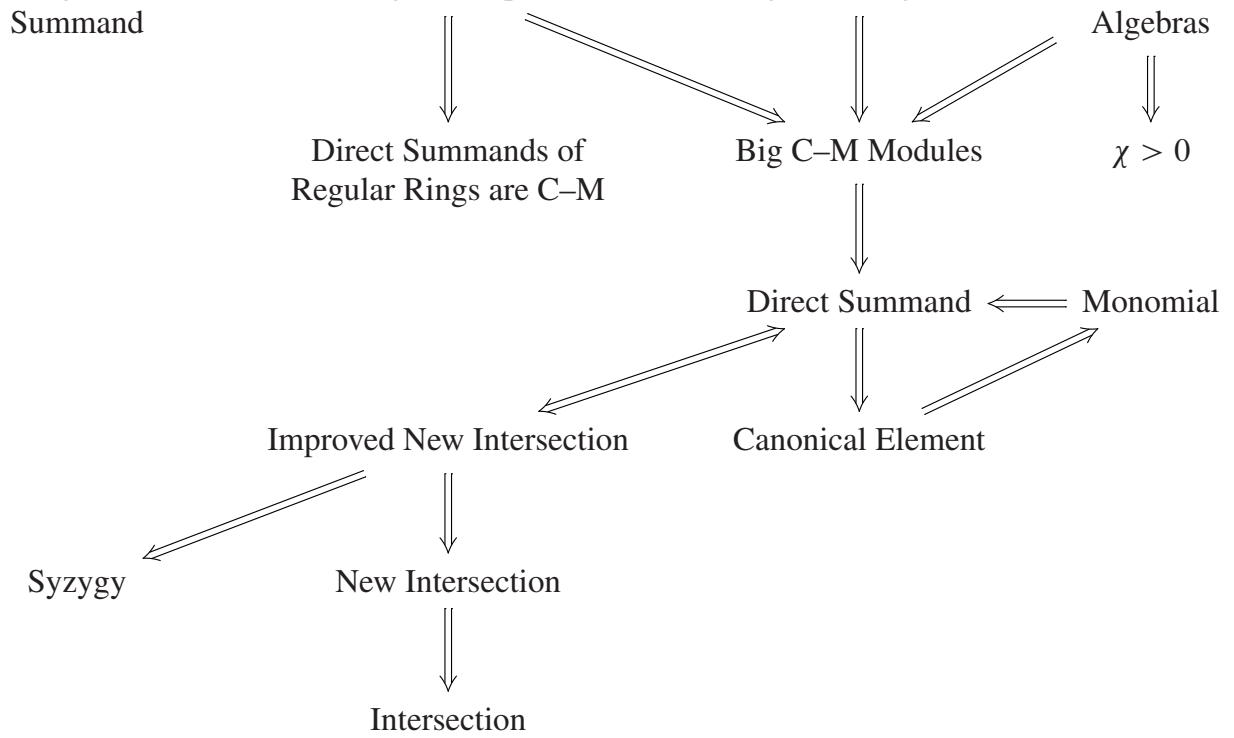

Small C-M

Figure 2. Hochster's diagram of homological conjectures in 2004.

\section{Almost Cohen-Macaulay Algebras}

As outlined above, Heitmann's proof of the Direct Summand Conjecture in dimension three introduced a new method for attacking many of the Homological Conjectures in mixed characteristic. In this section we will go into more detail about this method and questions that it raised.

What Heitmann showed originally was that if $A$ is a complete normal local domain of dimension 3 and of mixed characteristic, if $p, x, y$ is a system of parameters, and if $a p^{N} \in(x, y)$ for some $a \in A$, then for any integer $n>0$, there is a finite extension $B$ of $A$ such that $a p^{1 / n} \in(x, y) B$. This implies that the local cohomology $H_{\mathfrak{m}}^{2}\left(A^{+}\right)$ is annihilated by $p^{1 / n}$ for all $n>0$, where, as usual, $A^{+}$is the integral closure of $A$ in the algebraic closure of its quotient field. In a later paper ([23]) he extended this to show that $p$ can be replaced by any $u$ in the maximal ideal of $A$, and, using the fact that the condition that $A$ is a normal domain of dimension 3 , so that $H_{\mathfrak{m}}^{2}(A)$ has finite length, it is easy to see that this implies that $H_{\mathrm{m}}^{2}\left(A^{+}\right)$is annihilated by the maximal ideal of $A^{+}$. Thus it is a vector space over the field $A^{+} / \mathfrak{m}_{A^{+}}$. It is still an open question whether it is actually zero. 
As we also described earlier, the result, for example, that if $a p^{N} \in(x, y)$ in $A$ then $a p^{1 / n} \in(x, y)$ in $A^{+}$can be stated by the fact that certain closure operations have the colon-capturing property in this case. The closure operations are full extended plus closure of Heitmann [23] and dagger closure of Hochster and Huneke [32]. While the fact that full extended plus closure has this property is a stronger result, for the remainder of this section we will only consider dagger closure, since the fact that it has the colon-capturing property is enough to prove, for example, the Direct Summand Conjecture. We describe this in more detail.

Let $A$ be a ring as above; we take a valuation $v$ on $A$ with values in the ordered abelian group $\mathbb{R}$ of real numbers, Then $v$ is a function from $A$ to $\mathbb{R} \cup\{\infty\}$ satisfying

(i) $v(a b)=v(a)+v(b)$ for $a, b \in A$.

(ii) $v(a+b) \geq \min \{v(a), v(b)\}$ for $a, b \in A$.

(iii) $v(a)=\infty$ if and only if $a=0$.

We will assume also that $v(a) \geq 0$ for $a \in A$ and that $v(a)>0$ for $a$ in the maximal ideal of $A$. The existence of such a valuation follows from standard facts on extensions of valuations, see for example Zariski-Samuel [66], Chapter VI.

If $I$ is an ideal of a local domain $A$ with a valuation $v$ satisfying the above properties, then $a$ is in the dagger closure $I^{\dagger}$ of $I$ if there exist elements $u \in A^{+}$of arbitrarily small positive order, with $u x \in a A^{+}$. It follows from Heitmann's result that in mixed characteristic in dimension three dagger closure has the colon-capturing property. It also follows that, still in dimension three, the local cohomology module $H_{\mathfrak{m}}^{2}\left(A^{+}\right)$is annihilated by arbitrarily small elements. To generalize this we make the following definitions.

We say that an $A$-module $M$ is almost zero with respect to $v$ if for all $m \in M$ and for all $\epsilon>0$, there exists an $a \in A$ with $v(a)<\epsilon$ and $a m=0$.

This terminology comes from a paper of Faltings [16], where he proves that certain local cohomology groups are almost zero. The topic of almost zero modules was developed in much more detail by Gabber and Ramero [17].

Definition 4. An $A$-algebra $B$ is almost Cohen-Macaulay if

(i) $H_{(x)}^{i}(B)$ is almost zero for $i=0, \ldots, d-1$.

(ii) $B /\left(x_{1}, \ldots, x_{d}\right) B$ is not almost zero.

An alternative definition of almost Cohen-Macaulay can be obtained by defining a sequence $x_{1}, \ldots, x_{d}$ to be almost regular if $\left\{a \mid a x_{i} \in\left(x_{1}, \ldots, x_{i-1}\right)\right\} /\left(x_{1}, \ldots, x_{i-1}\right)$ is almost zero for $i=1, \ldots, d$ and defining $A$ to be almost Cohen-Macaulay if a system of parameters is almost regular (together with condition (2)). Standard methods show that this definition implies the former one (see for example Matsumura [44], Theorem 16.5 (i)). 
Question 1. Let $A$ be a complete Noetherian local domain. Is $A^{+}$almost CohenMacaulay?

Of course, the result of Hochster-Huneke and Huneke-Lyubeznik that we referred to above says that if $A$ has positive characteristic, then $A^{+}$is actually CohenMacaulay. However, this is not true in characteristic zero, since if we have a normal non-Cohen-Macaulay domain $A$, since $A$ is a direct summand of every finite extension using the trace map, a nontrivial element of local cohomology cannot go to zero in $A^{+}$. There is little evidence that this would be true in general, but there are some examples in characteristic zero in dimension 3 by Roberts, Singh, and Srinivas [59], and Heitmann, as we have seen, showed that it is true in mixed characteristic in dimension 3. As we have said, it is still open whether $A^{+}$is Cohen-Macaulay in that case.

This question can also be generalized further. Instead of the class of almost zero modules defined above, we can take other classes. To make the theory work we should take a class $\varphi$ of almost zero modules satisfying the following conditions.

(i) If $0 \rightarrow M^{\prime} \rightarrow M \rightarrow M^{\prime \prime} \rightarrow 0$ is a short exact sequence, then $M \in \mathcal{C}$ if and only if $M^{\prime}$ and $M^{\prime \prime}$ are in $e$.

(ii) $\zeta$ is closed under direct limits.

Question 2. Let $A$ be a local ring. Does there exist an almost Cohen-Macaulay algebra over $A$ for some class of almost zero modules?

\section{A Summary of Open Questions}

We summarize some of the main questions which remain open. Since they have varying degrees of likelihood of being true, we simply label them all as "Questions".

\subsection{The Serre Positivity Conjecture}

Question 3. Let $R$ be a ramified regular local ring of mixed characteristic, and let $M$ and $N$ be $R$-modules such that $M \otimes_{R} N$ has finite length. If $\operatorname{dim} M+\operatorname{dim} N=\operatorname{dim} R$, is $\chi(M, N)>0$ ?

This conjecture would follow from the existence of small Cohen-Macaulay modules. There has been some recent work to attempt to use Gabber's proof of the Nonnegativity Conjecture to prove this, but so far it has not been successful.

\subsection{Partial Euler Characteristics}

Question 4. If $R$ is a ramified regular local ring of mixed characteristic of dimension $d$ and $M$ and $N$ are $R$-modules such that $M \otimes_{R} N$ has finite length, is

$$
\chi_{i}(M, N)=\sum_{j=i}^{d}(-1)^{i+j} \text { length }(\operatorname{Tor}(M, N)) \geq 0 ?
$$




\subsection{Strong Multiplicity Conjectures}

Question 5. Let $A$ be a local ring, and let $M$ and $N$ be $A$-modules such that $M \otimes_{A} N$ has finite length and $M$ has finite projective dimension. Is $\operatorname{dim}(M)+\operatorname{dim}(N) \leq$ $\operatorname{dim}(A)$ ?

Question 6. Let $A$ be a local ring, and let $M$ and $N$ be $A$-modules such that $M \otimes_{A} N$ has finite length and both $M$ and $N$ have finite projective dimension.

(i) If $\operatorname{dim}(M)+\operatorname{dim}(N)<\operatorname{dim} A$, is $\chi(M, N)=0$ ?

(ii) If $\operatorname{dim}(M)+\operatorname{dim}(N)=\operatorname{dim} A$, is $\chi(M, N)>0$ ?

This question has been studied over the years, although it is still very much open. There are complexes which define positive cycles for which the positivity part fails, which may be a sign that the positivity is not true in this generality.

\subsection{Cohen-Macaulay Modules and Related Conjectures}

Question 7 (Small Cohen-Macaulay modules). Let $A$ be a complete local domain of dimension $d$. Does there exist a finitely generated $A$-module of depth $d$ ?

No one has yet succeeded in coming up with a way to approach this question in dimension 3 or greater.

Question 8 (Big Cohen-Macaulay modules). Let $A$ be a local domain of mixed characteristic of dimension $d$ with system of parameters $x_{1}, \ldots, x_{d}$. Does there exist an $A$-module $M$ for which

(i) $x_{1}, \ldots, x_{d}$ is a regular sequence on $M$.

(ii) $M /\left(x_{1}, \ldots, x_{d}\right) M \neq 0$.

As discussed at length, there are numerous conjectures which follow from this one, many of which are equivalent. Out of these we will state two, one because it is quite concrete, and the other because it is the strongest of these conjectures. Both of these are open in the case where $A$ has mixed characteristic and dimension greater than three.

Question 9 (Monomial Conjecture). Let $A$ be a local ring with system of parameters $x_{1}, \ldots, x_{d}$. Is $x_{1}^{t} x_{2}^{t} \cdots x_{d}^{t}$ in the ideal $\left(x_{1}^{t+1}, x_{2}^{t+1}, \ldots, x_{d}^{t+1}\right)$ ?

Question 10. Can one construct weakly functorial big Cohen-Macaulay algebras?

We refer to Section 6.1 for a precise statement of what this means. 


\subsection{Almost Cohen-Macaulay Algebras}

Question 11. Let $A$ be a local ring. Does $A$ have an almost Cohen-Macaulay algebra?

We refer to the previous section for a precise statement of this question.

\section{Bibliography}

[1] Auslander, M., Modules over unramified regular local rings. Proceedings, International Congress Stockholm; 1962. p. 230-233.

[2] Avramov, L., Buchweitz, R., Iyengar, S., Class and rank of differential modules. Invent Math. 2007;169:1-35.

[3] Berthelot, P., Altérations de variétés algébriques [d'après A. J.de Jong]. Sémin Bourbaki. 1996:exposé 815.

[4] Bertin, M.-J., Anneaux d'invariants d'anneaux de polynomes, en caractéristique $p$. C R Acad Sci Paris Sér A-B 1967;264:A653-A656.

[5] Brodman, M. P., Sharp, R. Y., Local cohomology: an algebraic introduction with geometric applications. vol. 60 of Cambridge studies in advanced mathematics. Cambridge: Cambridge University Press; 1998.

[6] Dao, H., Decency and Tor-rigidity for modules over hypersurfaces. arXiv: math.AC/0611568.

[7] Dutta, S. P., On the canonical element conjecture. Trans Amer Math Soc. 1987;299:803811.

[8] Dutta, S. P., A note on the monomial conjecture. Trans Amer Math Soc. 1998;350:28712878.

[9] Dutta, S. P., On negativity of higher Euler characteristics. Amer J Math. 2004;126:13411354.

[10] Dutta, S. P., Intersection multiplicity of Serre on regular schemes. J Algebra. 2008;319: $1530-1554$.

[11] Dutta, S. P., Hochster, M., McLaughlin, J. E., Modules of finite projective dimension with negative intersection multiplicities. Invent Math. 1985;79:253-291.

[12] Eisenbud, D., Homological algebra on a complete intersection, with an application to group representations. Trans Amer Math Soc. 1980;260:35-64.

[13] Evans, E. G., Griffith, P., The syzygy problem. Ann of Math (2). 1981;114:323-333.

[14] Evans, E. G., Griffith, P., Syzygies. vol. 106 of London Mathematical Society lecture note series. Cambridge: Cambridge University Press; 1985.

[15] Evans, E. G., Griffith, P., A graded syzygy theorem in mixed characteristic. Math Res Lett. 2001;8:605-611.

[16] Faltings, G., Almost étale extensions. Astérisque 2002;297:185-270. 
[17] Gabber, O., Ramero, L., Almost ring theory, vol. 1800 of Lecture notes. Springer.

[18] Gillet, H., Soulé, C. K-théorie et nullité des multiplicités d'intersection. C R Acad Sci Paris Sér I. 1985;300(3):71-74.

[19] Gillet, H., Soulé, C., Intersection theory using Adams operations. Invent Math. 1987;90: 243-277.

[20] Heitmann, R., A counterexample to the rigidity conjecture for rings. Bull. Amer Math Soc (N S). 1993;29:94-97.

[21] Heitmann, R., The plus closure in mixed characteristic. J Algebra. 1997;193:688-708.

[22] Heitmann, R. C., The direct summand conjecture in dimension three. Ann of Math (2). 2002;156:695-712.

[23] Heitmann, R. C., Extended plus closure and colon-capturing. J Algebra. 2005;293:407426.

[24] Hochster, M., Topics in the homological theory of modules over commutative rings. vol. 24 of CBMS regional conference series in mathematics. 1975.

[25] Hochster, M., Canonical elements in local cohomology modules and the direct summand conjecture. J Algebra. 1983;84:503-553.

[26] Hochster, M., Euler characteristics over unramified regular local rings. Illinois J Math. 1984;28:281-285.

[27] Hochster, M., Nonnegativity of intersection multiplicities in ramified regular local rings following Gabber/De Jong/Berthelot [unpublished notes]. Available from: http://www. math.lsa.umich.edu/ hochster/mult.ps.

[28] Hochster, M., Big Cohen-Macaulay algebras in dimension three via Heitmann's theorem. J Algebra. 2002;254:395-408.

[29] Hochster, M., Notes from the 2004 Microprogram at the University of Utah. Available from: http://www.math.utah.edu/vigre/minicourses/algebra/hochster.pdf.

[30] Hochster, M., Homological conjectures, old and new. Illinois J Math. 2007;51(1):151169.

[31] Hochster, M., Huneke, C., Tight closure, invariant theory, and the Briançon-Skoda theorem. J Amer Math Soc. 1990;3:31-116.

[32] Hochster, M., Huneke, C., Tight closure and elements of small order in integral extensions. J Pure Appl Algebra. 1991;71:233-247.

[33] Hochster, M., Huneke, C., Infinite integral extensions and big Cohen-Macaulay algebras. Ann of Math (2). 1992;135:53-89.

[34] Hochster, M., Huneke, C., Applications of the existence of big Cohen-Macaulay algebras. Adv Math. 1995;113:45-117.

[35] Huneke, C., Tight closure and its applications. vol. 88 of CBMS regional conference series in mathematics. 1996.

[36] Huneke, C., Lyubeznik, G., Absolute integral closure in positive characteristic. Adv Math. 2007;210:498-504. 
[37] Huneke, C., Wiegand, R., Tensor products of modules and the rigidity of Tor. Math Ann. 1994;299:449-476.

[38] Iyengar, S., Leuschke, G., Leykin, A., Miller, C., Miller, E., Singh, A., Walther, U., Twenty-four hours of local cohomology. vol. 87 of graduate studies in mathematics. Providence, RI: American Mathematical Society; 2007.

[39] de Jong, A. J., Smoothness, semi-stability, and alterations. Publ Math Inst Hautes Études Sci. 1996;83:51-93.

[40] Katz, D., On the existence of maximal Cohen-Macaulay modules over $p$ th root extensions. Proc Amer Math Soc. 1999;127:2601-2609.

[41] Kurano, K., A remark on the Riemann-Roch formula on affine schemes associated with Noetherian local rings. J Math Soc Japan 1993;45:369-390.

[42] Kurano, K., Roberts, P., The positivity of intersection multiplicities and symbolic powers of prime ideals. Compos Math. 2000;122:165-182.

[43] Lichtenbaum, S., On the vanishing of Tor in regular local rings. Illinois J Math. 1966;10: 220-226.

[44] Matsumura, H., Commutative rings. Cambridge University Press; 1986.

[45] McCullough, J., A note on the strong direct summand conjecture. Proc Amer Math Soc 2009;127:2857-2864.

[46] Miller, C., Singh, A., Intersection multiplicities over Gorenstein rings. Math Ann. 2000; 317:155-171.

[47] Peskine, C., Szpiro, L., Dimension projective finie et cohomologie locale. Publ Math Inst Hautes Études Sci. 1973;42:49-119.

[48] Peskine, C., Szpiro, L., Syzygies et multiplicités. C R Acad Sci Paris Sér A 1974;278: $1421-1424$.

[49] Ranganathan, N., Splitting in module-finite extensions and the vanishing conjecture for maps of Tor [thesis]. University of Michigan; 2000.

[50] Roberts, P., Two applications of dualizing complexes over local rings. Ann Sci Éc Norm Supér (4). 1976;9:103-106.

[51] Roberts, P., The vanishing of intersection multiplicities of perfect complexes. Bull Amer Math Soc. 1985;13:127-130.

[52] Roberts, P., Le théorème d'intersection. C R Acad Sci Paris Sér I. 1987;304(7):177-180.

[53] Roberts, P., Intersection theorems. In: Commutative Algebra. Proceedings of an MSRI Microprogram. New York: Springer-Verlag; 1989. p. 417-436.

[54] Roberts, P., Negative intersection multiplicities on singular varieties. Contemp Math. 1991;123(Proceedings of the Zeuthen Conference 1989):213-222.

[55] Roberts, P., The homological conjectures. In: Free resolutions in commutative algebra and algebraic geometry. Proceedings, Sundance 90. vol. 2 of Research notes in mathematics. 1992. 
[56] Roberts, P., Multiplicities and Chern classes in local algebra. vol. 133 of Tracts in mathematics. Cambridge University Press; 1998.

[57] Roberts, P., Recent developments on Serre's multiplicity conjectures: Gabber's proof of the nonnegativity conjecture. Enseign Math (2). 1998;44:305-324.

[58] Roberts, P., Singh, A., Reconciling Riemann-Roch results. To appear.

[59] Roberts, P., Singh, A., Srinivas, V., Annihilators of local cohomology in characteristic zero. Illinois J Math. 2007;51:237-254.

[60] Roberts, P., Srinivas, V., Modules of finite length and finite projective dimension. Invent Math. 2003;151:1-27.

[61] Sather-Wagstaff, S., A dimension inequality for Cohen-Macaulay rings. Trans Amer Math Soc. 2002;354:993-1005.

[62] Serre, J.-P., Local algebra. Springer monographs in mathematics, Berlin: SpringerVerlag; 2000.

[63] Strooker, J. R., Homological questions in commutative algebra. vol. 106 of London Mathematical Society lecture note series. Cambridge: Cambridge University Press; 1990.

[64] Strooker, J. R., Stückrad, J., Monomial conjecture and complete intersections. Manuscripta Math. 1993;79:153-159.

[65] Szpiro, L., Sur la théorie des complexes parfaits. In: Commutative Algebra. Proceedings, Durham 1981. vol. 72 of London Mathematical Society lecture notes series. 1982. 83-90.

[66] Zariski, O., Samuel, P., Commutative algebra II. vol. 29 of Graduate texts in mathematics. New York, Heidelberg: Springer-Verlag; 1975.

\section{Author Information}

Paul C. Roberts, Department of Mathematics, University of Utah, Salt Lake City, UT, USA.

E-mail: roberts@math.utah.edu 